\title{
Printed, Soft, Nanostructured Strain Sensors for Monitoring of Structural Health and Human Physiology
}

Robert Herbert, $\uparrow$ Hyo-Ryoung Lim, $\dagger$ and Woon-Hong Yeo* $, \dagger, \S, \$$

$\dagger$ George W. Woodruff School of Mechanical Engineering, Institute for Electronics and Nanotechnology, Georgia Institute of Technology, Atlanta, GA 30332, United States

$\$$ Wallace H. Coulter Department of Biomedical Engineering, Georgia Institute of Technology, Atlanta, GA 30332, United States

$\S$ Parker H. Petit Institute for Bioengineering and Biosciences, Neural Engineering Center, Institute for Materials, Institute for Robotics and Intelligent Machines, Georgia Institute of Technology, Atlanta, Georgia 30332, United States

*Address correspondence to whyeo@gatech.edu 


\section{Note S1. Fabrication of printed strain sensors}

1. Prepare glass slide with sacrificial PMMA layer.

a. Clean glass slide by rinsing surfaces with acetone followed by isopropyl alcohol. Dry with compressed $\mathrm{N}_{2}$.

b. Spin-coat PMMA onto the cleaned glass slide at $3000 \mathrm{RPM}$ for $30 \mathrm{~s}$.

c. Bake for 3 minutes at $180{ }^{\circ} \mathrm{C}$.

2. Create ink mixtures for printing.

a. Polyimide ink: Mix PI-2545 precursor (HD MicroSystems) with the solvent NMP (Sigma-Aldrich) in a 4:1 ratio.

b. AgNW ink: Mix AgNW solution (0475NW6I, Nanostructured \& Amorphous Materials, Inc.) with IPA in a 2:1 ratio.

3. Print bottom polyimide layer.

a. Set stage temperature to $80{ }^{\circ} \mathrm{C}$ to heat substrate during printing.

b. Load polyimide ink into the pneumatic atomizer and print 4 passes at a speed of $10 \mathrm{~mm} / \mathrm{s}$ with a $300 \mu \mathrm{m}$ nozzle diameter.

c. Cure polyimide layer in an oven for 1 hour at $240{ }^{\circ} \mathrm{C}$.

4. Print AgNW layer.

a. Set stage temperature to $80{ }^{\circ} \mathrm{C}$ to heat substrate during printing.

b. Plasma treat printed polyimide for 30 second to enhance adhesion of AgNWs to polyimide surface.

c. Load AgNW ink into the ultrasonic atomizer. Ink atomization is performed in the ultrasonic atomizer with a water bath temperature of $27^{\circ} \mathrm{C}$ and atomizer current of $0.6 \mathrm{~mA}$. Nozzle is aligned with printed polyimide and then prints desired number of passes at a speed of $2 \mathrm{~mm} / \mathrm{s}$ with a $200 \mu \mathrm{m}$ nozzle diameter.

5. Print top polyimide layer.

a. Set stage temperature to $80{ }^{\circ} \mathrm{C}$ to heat substrate during printing.

b. Load polyimide ink into the pneumatic atomizer and print 4 passes at a speed of $10 \mathrm{~mm} / \mathrm{s}$ with a $300 \mu \mathrm{m}$ nozzle diameter.

c. Cure polyimide layer in an oven for 1 hour at $240{ }^{\circ} \mathrm{C}$.

6. Dissolve PMMA layer and transfer sensors to water soluble tape.

a. Cover the printed sensors on the glass slide with a piece of Texwipe. Clamp a second glass slide on top of the printed sensors and Texwipe to hold the sensors in place when dissolving PMMA.

b. Submerge the glass slides with Texwipe into acetone for 1.5 hours.

c. Remove slides from acetone, unclamp secondary glass slide, and remove Texwipe. This leaves the initial glass slide with printed sensors still on its surface.

d. Apply the adhesive side of water soluble tape (ASWT-2, Aquasol) to the glass slide with sensors. Press the tape firmly onto the slide.

e. Gently peel tape off of the glass slide and the sensors will stick to the tape.

7. Prepare elastomer substrates. These elastomer substrates are the 'adhesive' elastomers that interface with skin or a surface.

a. Elastomers were mixed by hand in plastic cups. The following ratios by weight for each elastomer are as follows:

i. Ecoflex 00-30 (Smooth-On): 1:1 ratio of Part A to Part B

ii. Ecoflex Gel (Smooth-On): 1:1 ratio of Part A to Part B

iii. PDMS (Sylgard 184, Dow): 10:1 ratio of Base to Cure 
iv. Silbione (Silbione RT Gel, Elkem): 1:1 ratio of Part A to Part B

v. For combinations of Ecoflex 30 and Gel, the two elastomers were mixed separately according to the above ratios and then combined in the $1: 1$ or $1: 2$ ratio.

b. A glass slide is prepared by taping a sheet of PVA to the top of it.

c. Elastomer is spin coated at 500 RPM for 30 seconds onto the PVA film.

d. To cure elastomers, the following conditions were used on a hot plate:

i. All Ecoflex mixtures: $60^{\circ} \mathrm{C}$ for 30 minutes

ii. PDMS: $80{ }^{\circ} \mathrm{C}$ for 1 hour

iii. Silbione: $60^{\circ} \mathrm{C}$ for 30 minutes

8. Transfer sensors from water soluble tape to elastomer substrate.

a. The adhesive side of the tape, with the printed sensors, is laminated onto the elastomer substrate.

b. A micropipette is used to cover the top surface of the tape with DI water. The water is left on the tape for a few minutes.

c. After a few minutes, the tape residue is washed off by gently running DI water over the sensors. Water may be added until tape residue is washed off.

d. The elastomer substrate with sensors is heated at $80^{\circ} \mathrm{C}$ for 10 minutes to dry.

9. Wiring sensors.

a. To wire the transferred sensors, flexible film cables (heat seal connectors, Elform) are cut into individual cables with an approximate length of $6 \mathrm{~cm}$.

b. A small amount of fast drying silver paint (16040-30, Ted Pella) is applied to the sensor contact pads with the tip of a stainless steel wire.

c. While the silver paint is wet, the flexible film cable is applied down onto the silver paint and contact pad. The wire is held in place for a few minutes until the silver paint dries.

10. Encapsulating the wired sensors. The top elastomer layer added to the below substrate and sensor is the 'support elastomer'.

a. To encapsulate the sensors, a small amount of elastomer (mixed according to the previous ratios) is added with a micropipette to the top of the wired sensor. The deposited elastomer is thinned with compressed $\mathrm{N}_{2}$.

b. Curing the encapsulation is performed with the previous conditions.

11. Remove completed sensors from glass slide and PVA film.

a. The printed sensor and two elastomer layers are cut into a square surrounding the sensor with a razor blade.

b. The cut samples are then removed from the PVA film by peeling the sensor from the film with tweezers.

12. Laminate sensors onto surface or skin.

a. To attach to a surface or skin, the sensors are placed onto the desired surface with the elastomer substrate ('adhesive elastomer') facing the surface. Pressing the sensor against the surface or skin so that all edges are in contact completes sensor attachment. The sensor is then ready for testing by attaching the flexible film cable ends to a multimeter with mini hook wire ends. 


\section{Note S2. Analytical model for conformal contact and contact pressure.}

The interfacial mechanics between skin and sensor is described as ${ }^{1-2}$ :

$$
U_{\text {conformal }}=U_{\text {bending }}+U_{\text {skin }}+U_{\text {adhesion }}
$$

where $U_{\text {bending }}$ is sensor bending energy, $U_{\text {skin }}$ is skin elastic energy, and $U_{\text {adhesion }}$ is contact adhesion energy. Conformal contact occurs when the adhesion energy is greater than the sum of bending and elastic energy.

To calculate each energy term, the skin surface is modeled as a sinusoidal surface:

$$
y(x)=\frac{h_{\text {rough }}}{2}\left(1+\cos \frac{2 \pi x}{\lambda_{\text {rough }}}\right)
$$

where $h_{\text {rough }}$ is amplitude and $\lambda_{\text {rough }}$ is wavelength. During conformal contact, the sensor matches the skin surface so that sensor displacement is:

and skin displacement is:

$$
w(x)=\frac{h}{2}\left(1+\cos \frac{2 \pi x}{\lambda_{\text {rough }}}\right)
$$

$$
u_{z}(x)=y-w=\frac{h_{\text {rough }}-h}{2}\left(1+\cos \frac{2 \pi x}{\lambda_{\text {rough }}}\right)
$$

The following parameters define the skin conditions:

$$
h_{\text {rough }}=55 \mathrm{um} \quad \lambda_{\text {rough }}=385 \mathrm{um} \quad E_{\text {skin }}=130 \mathrm{kPa}
$$

With this defined, bending energy is given as:

$$
U_{\text {bending }}=\frac{1}{\lambda_{\text {rough }}} \int_{0}^{\lambda_{\text {rough }}} \frac{E I_{\text {sensor }}\left(w^{\prime \prime}\right)^{2}}{2} d x=\frac{\pi^{4} E I_{\text {sensor }} h^{2}}{\lambda_{\text {rough }}^{4}}
$$

where the bending stiffness is calculated as a multilayered structure of elastomer, polyimide, and a polyimide- $\mathrm{AgNW}$ composite:

$$
E I_{\text {sensor }}=\alpha E I_{P I / A g N W}+(1-\alpha) E I_{\text {elastomer }}
$$

Here, $\alpha$ is the areal fraction of polyimide. Each bending stiffness is defined as:

$$
\begin{gathered}
E I_{\text {elastomer }}=E_{\text {elastomer }} h_{\text {elastomer }}{ }^{3} / 12 \\
E I_{P I / A g N W}=\sum_{i=1}^{N} E_{i} h_{i}\left[\left(b-\sum_{j=1}^{i} h_{j}\right)^{2}+\left(b-\sum_{j=1}^{i} h_{j}\right) h_{i}+\frac{1}{3} h_{i}^{2}\right] \\
b=\sum_{i=1}^{N} E_{i} h_{i}\left(\sum_{j=1}^{i} h_{j}-\frac{1}{2} h_{i}\right) / \sum_{i=1}^{N} E_{i} h_{i}
\end{gathered}
$$

Here, the sensor is assumed to be a layer of polyimide and a layer of composite polyimide and AgNW. $N$ is equal to 3 to account for the two sensor layers and encapsulation. Elastomer modulus $(47.2 \mathrm{kPa})$ and thickness $(300 \mu \mathrm{m})$ is $E_{\text {elastomer }}$ and $h_{\text {elastomer }}$ while the polyimide and AgNW layer is given by $E_{i}$ and $h_{i}$. Polyimide modulus is defined as $2.5 \mathrm{GPa}$, polyimide thickness is $3.5 \mu \mathrm{m}$. AgNW modulus is $140 \mathrm{GPa}^{3}$ and the composite AgNW/PI layer thickness is $5 \mu \mathrm{m}$. To calculate the modulus of the composite AgNW and polyimide layer, the Voigt model is used $^{4-5}$ :

$$
E_{P I / A g N W}=E_{P I}\left(1-V_{A g N W}\right)+E_{A g N W} V_{A g N W}
$$

where $E_{P I}$ and $E_{A g N W}$ are the modulus of polyimide and $\mathrm{AgNW}$, and $V_{A g N W}$ is the volume fraction of AgNW. Volume fill fraction of AgNWs is assumed to be 50\%, which is expected to be an overestimate considering 4 passes of PI is $3.5 \mu \mathrm{m}$ and the total AgNW/PI composite height is 5 $\mu \mathrm{m}$. The Voigt model estimates the upper bounds of a composite structure where the nanowires are aligned in the same direction as the applied load. If loading is applied perpendicular to the nanowires, the Reuss model may be used ${ }^{4-5}$ :

$$
\frac{1}{E_{P I / A g N W}}=\frac{V_{A g N W}}{E_{A g N W}}+\frac{1-V_{A g N W}}{E_{P I}}
$$


This model provides the lower bound of modulus for a composite structure. Randomly oriented networks generally are closer to the lower bound for modulus ${ }^{5}$. However, in this work the upper bound is used to confirm conformal contact and indicate a maximum possible bending stiffness.

Skin elastic energy is given by:

Where the normal stress is:

$$
U_{\text {skin }}=\frac{1}{\lambda_{\text {rough }}} \int_{0}^{\lambda_{\text {rough }}} \frac{\sigma_{z} u_{z}}{2} d x=\frac{\pi E_{\text {skin }}\left(h_{\text {rough }}-h\right)^{2}}{16 \lambda_{\text {rough }}}
$$

Then the adhesion energy is:

$$
\sigma_{z}=\frac{\pi E_{\text {skin }}\left(h_{\text {rough }}-h\right)}{2 \lambda_{\text {rough }}} \cos \frac{2 \pi x}{\lambda_{\text {rough }}}
$$

$$
U_{\text {adhesion }}=-\gamma \int_{0}^{\lambda_{\text {rough }}} \sqrt{1+\left(w^{\prime}\right)^{2}} d x \approx-\gamma\left(1+\frac{\pi^{2} h^{2}}{4 \lambda_{\text {rough }}^{2}}\right)
$$

Elastomer work of adhesion $(\gamma)$ is based on our previous study ${ }^{1}$.

Minimizing the total energy yields a maximum sensor displacement of:

$$
h=\frac{E_{\text {skin }} h_{\text {rough }}}{\frac{16 \pi^{3} E I_{E E S}}{\lambda_{\text {rough }}^{3}}+E_{\text {skin }}}
$$




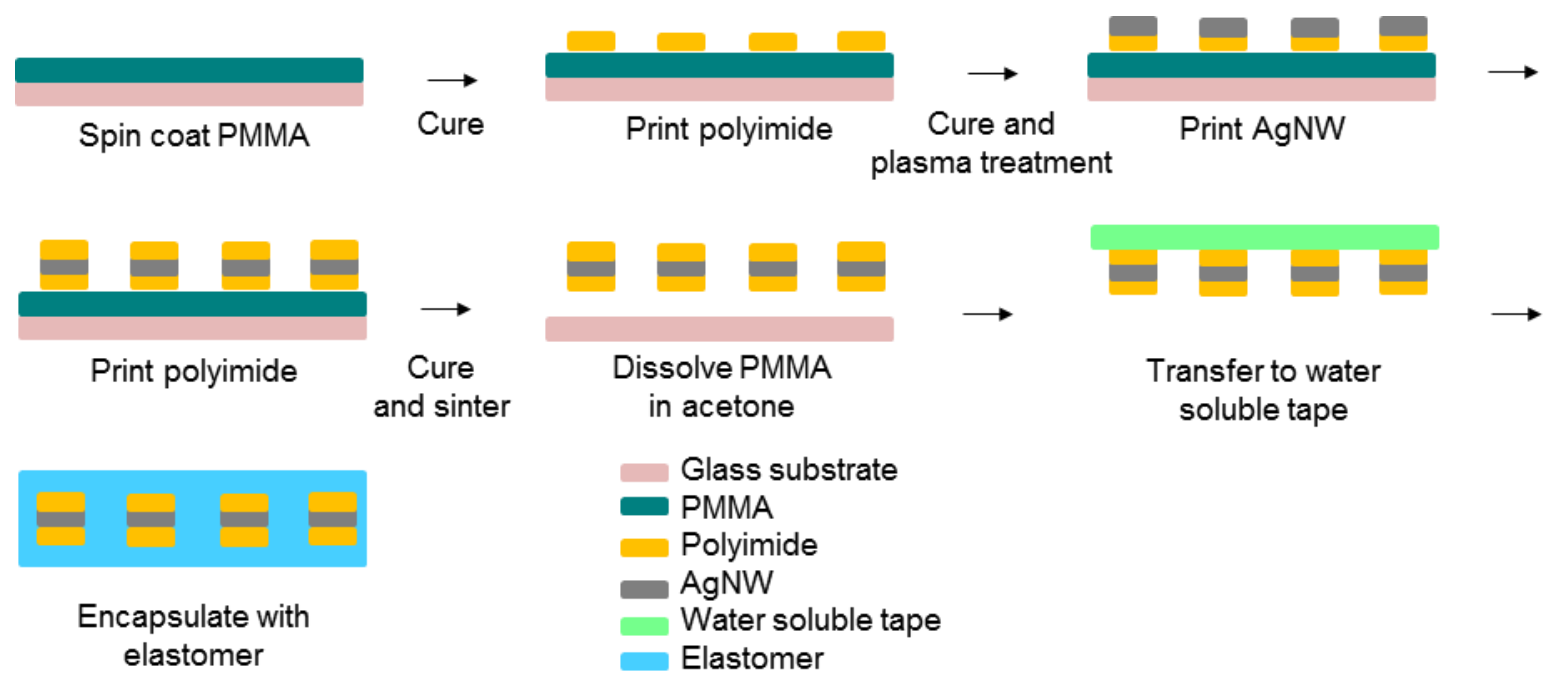

Figure S1. Fabrication steps for printed strain sensors. 
A

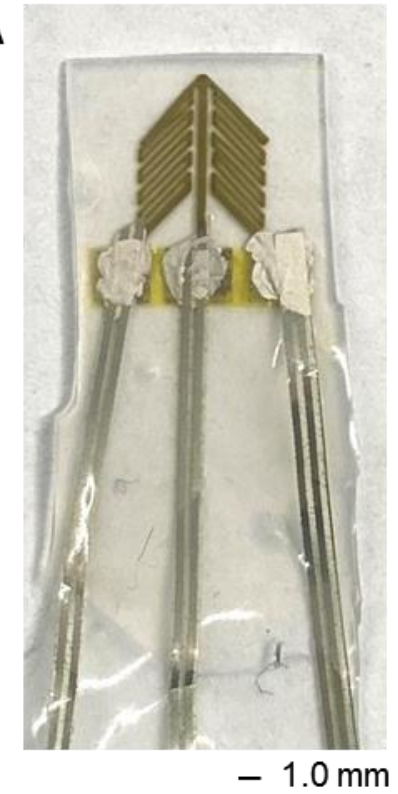

B

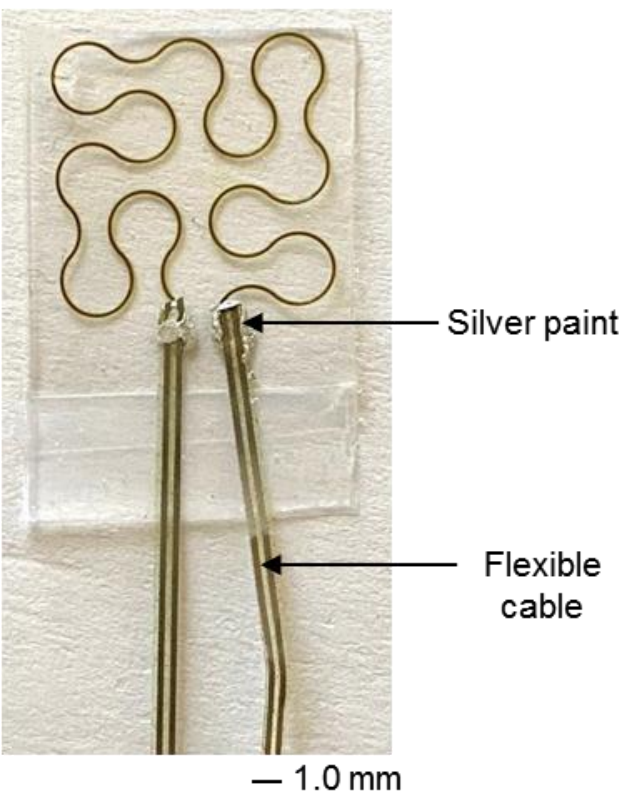

Figure S2. Strain sensor wiring using silver paint to attach flexible film cables to (A) structural sensor and (B) human health monitoring sensor. 

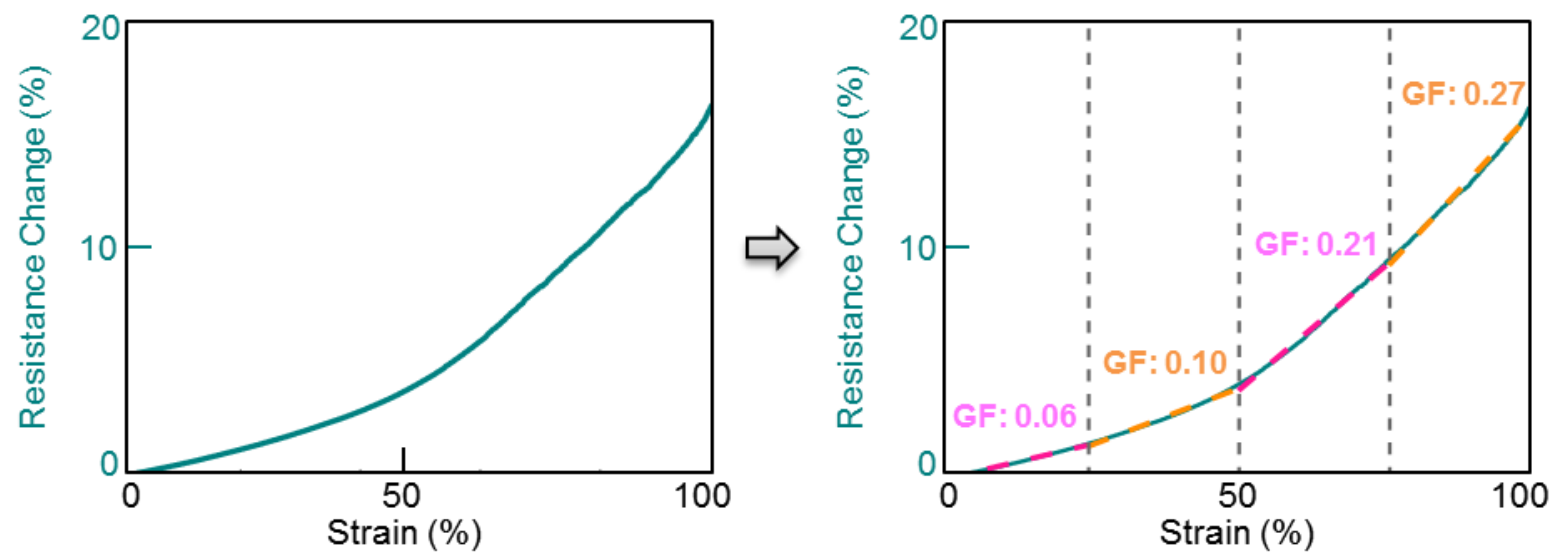

Figure S3. Illustration of GF calculation. The resistance curve is segmented into $25 \%$ strain regions and the slope within each region is calculated. This slope is the GF. Regions between 0 to $25 \%, 25 \%$ to $50 \%, 50 \%$ and $75 \%$, and $75 \%$ to $100 \%$ are shown. 


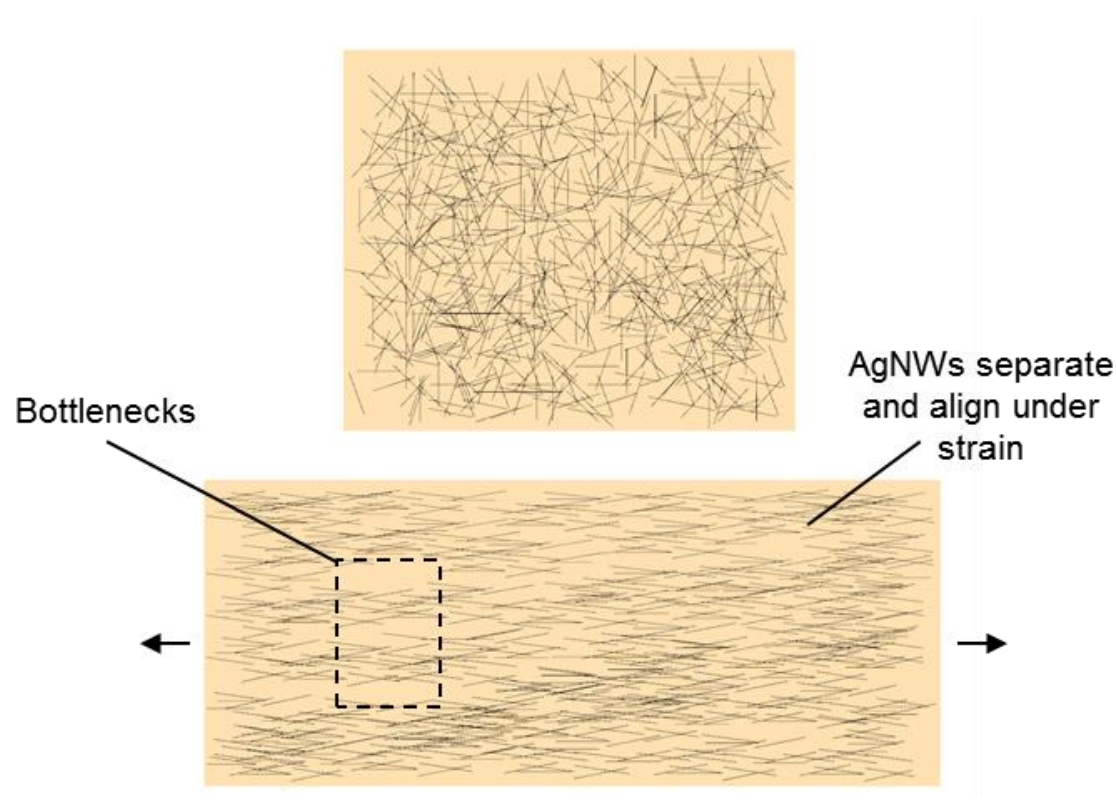

Figure S4. Illustration of strain sensing mechanism. During stretching, the AgNWs separate and align in the direction of stretching. This reduces electrical connections and increases resistance. Additionally, bottlenecks may form in the AgNW network which creates a nonlinear response in resistance. 

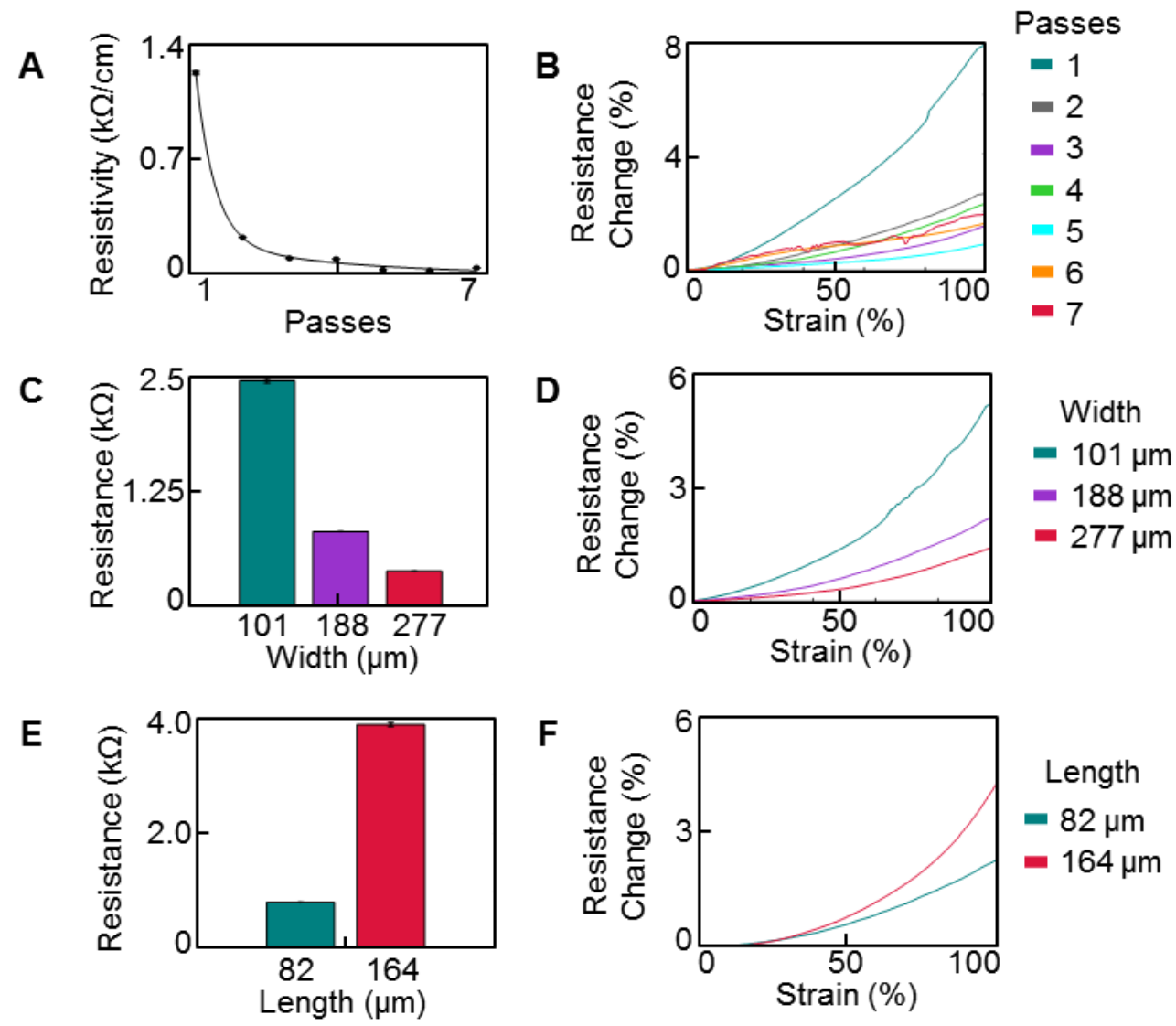

Figure S5. Resistance change with strain for printed strain sensor designs. (A) AgNW resistivity for different number of AgNW print passes. (B) Resistance changes for sensors with varying number of $\mathrm{AgNW}$ passes. (C) Sensor resistance and (D) resistance changes for different trace widths. (E) Sensor resistances and (F) resistance changes for two lengths of sensors. 

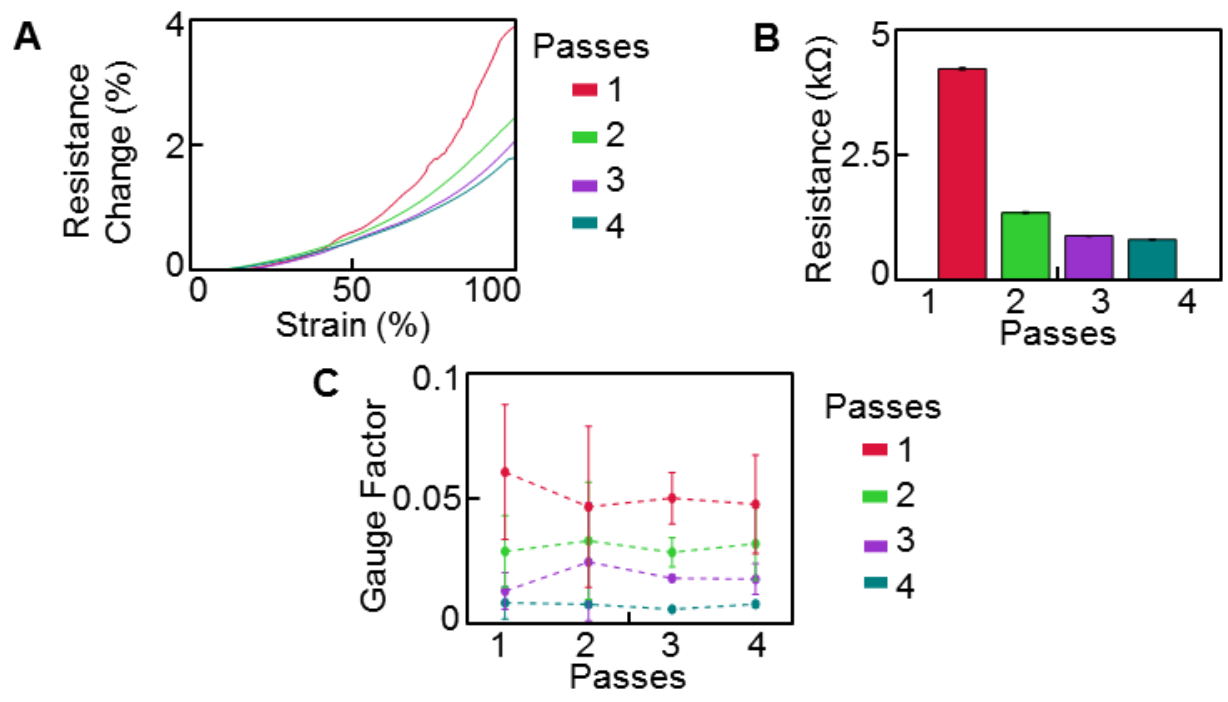

$$
\begin{gathered}
\text { Passes } \\
=1 \\
=2 \\
=3 \\
=4
\end{gathered}
$$

Figure S6. Gauge factor tuning via passes for small unit cell sensor. (A) Resistance changes, (B) sensor resistance, and (C) gauge factors for small unit cell sensors with 1 to 4 AgNW passes. 


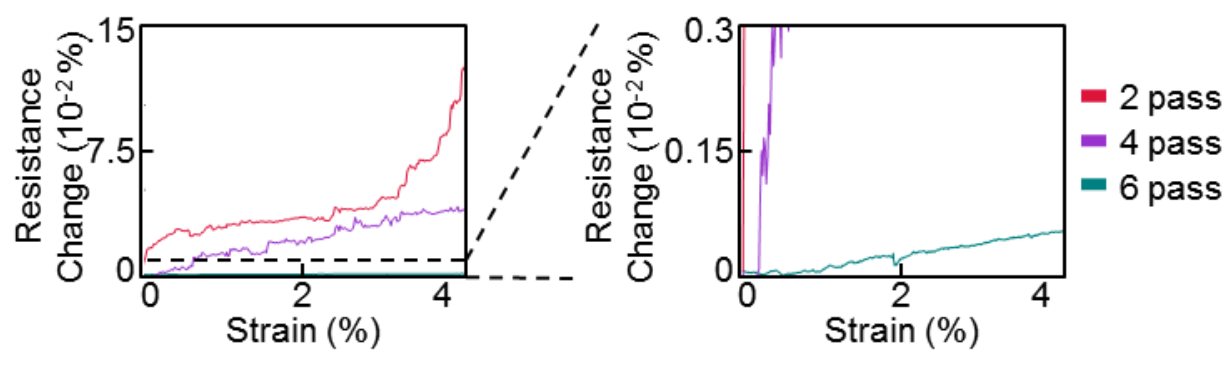

Figure S7. Resistance change with strain for flexible sensor with different number of passes. 

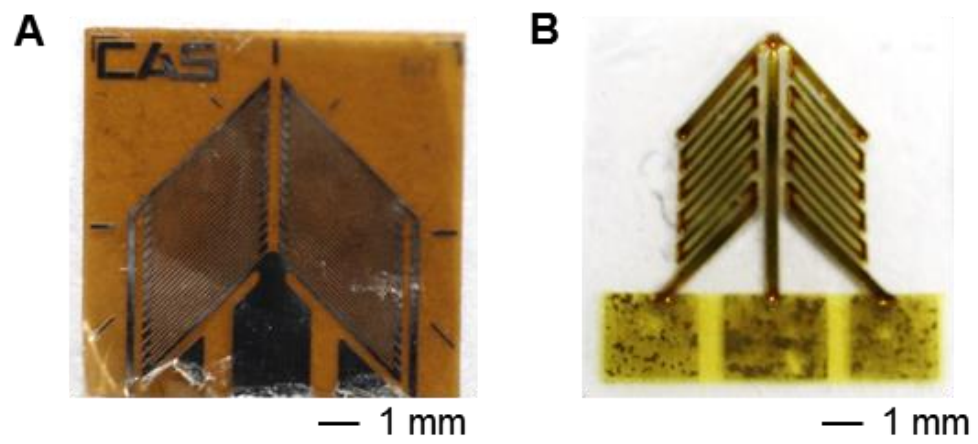

Figure S8. Comparison of two strain sensors. Photos of (A) a commercial strain sensor and (B) a printed, soft strain sensor, both used for adhesion tests. 

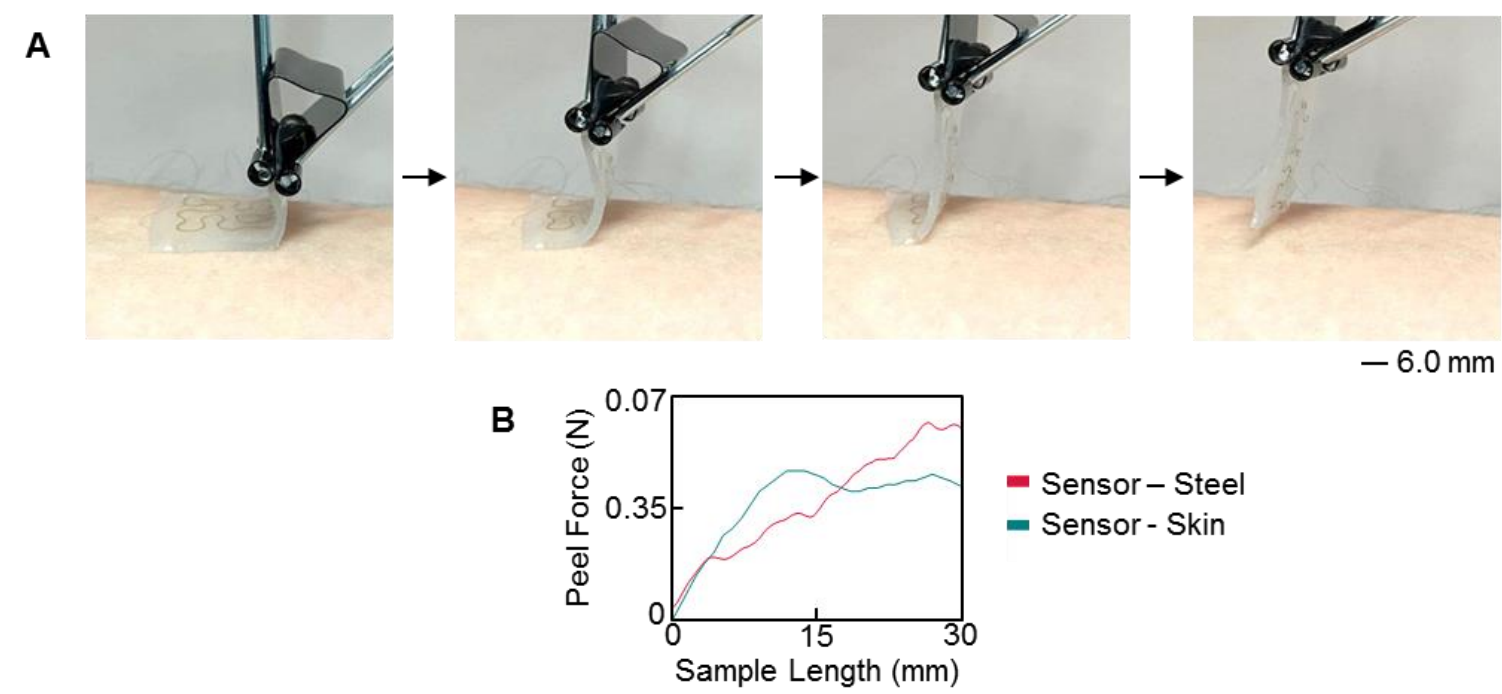

Figure S9. Adhesion between sensor and skin is demonstrated with a peel test after laminating sensor to skin. (A) Photos of peeling sensor from skin. (B) Peel force is shown for peel tests from a steel surface and from skin. 

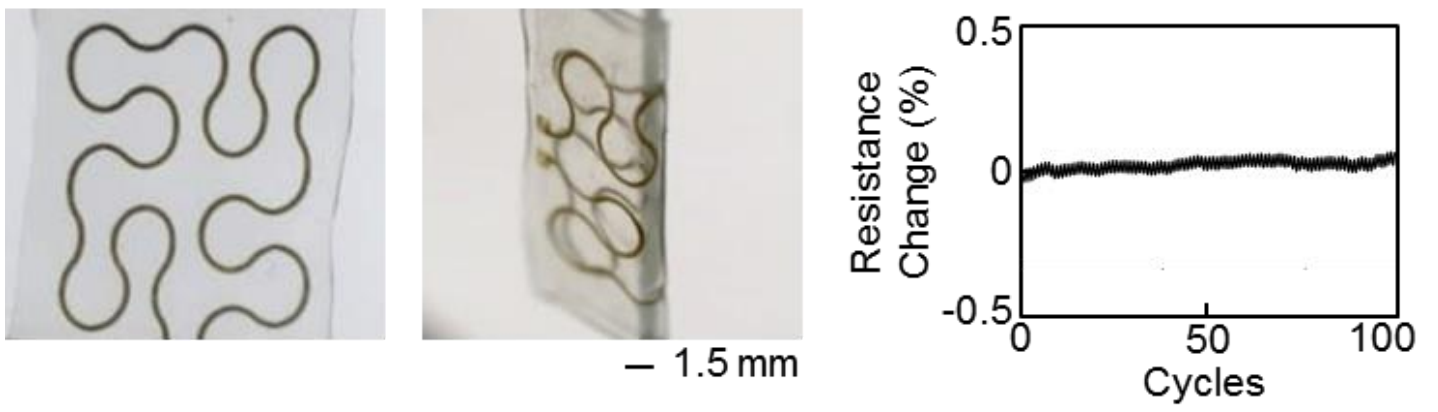

Figure S10. Cyclic bending result of a printed stretchable sensor. 
A

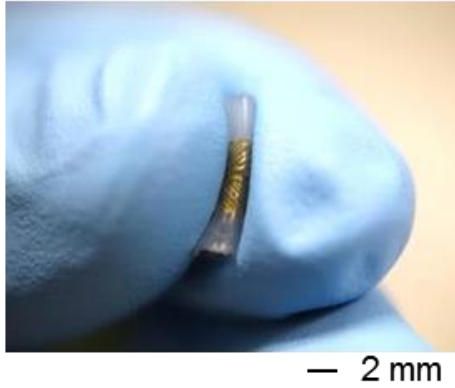

B
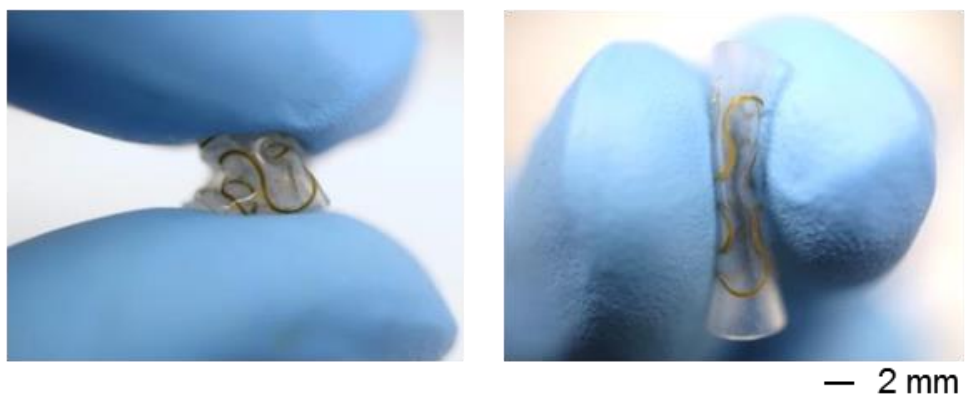

Figure S11. Large deformations of (A) flexible strain sensor and (B) wearable strain sensor. 

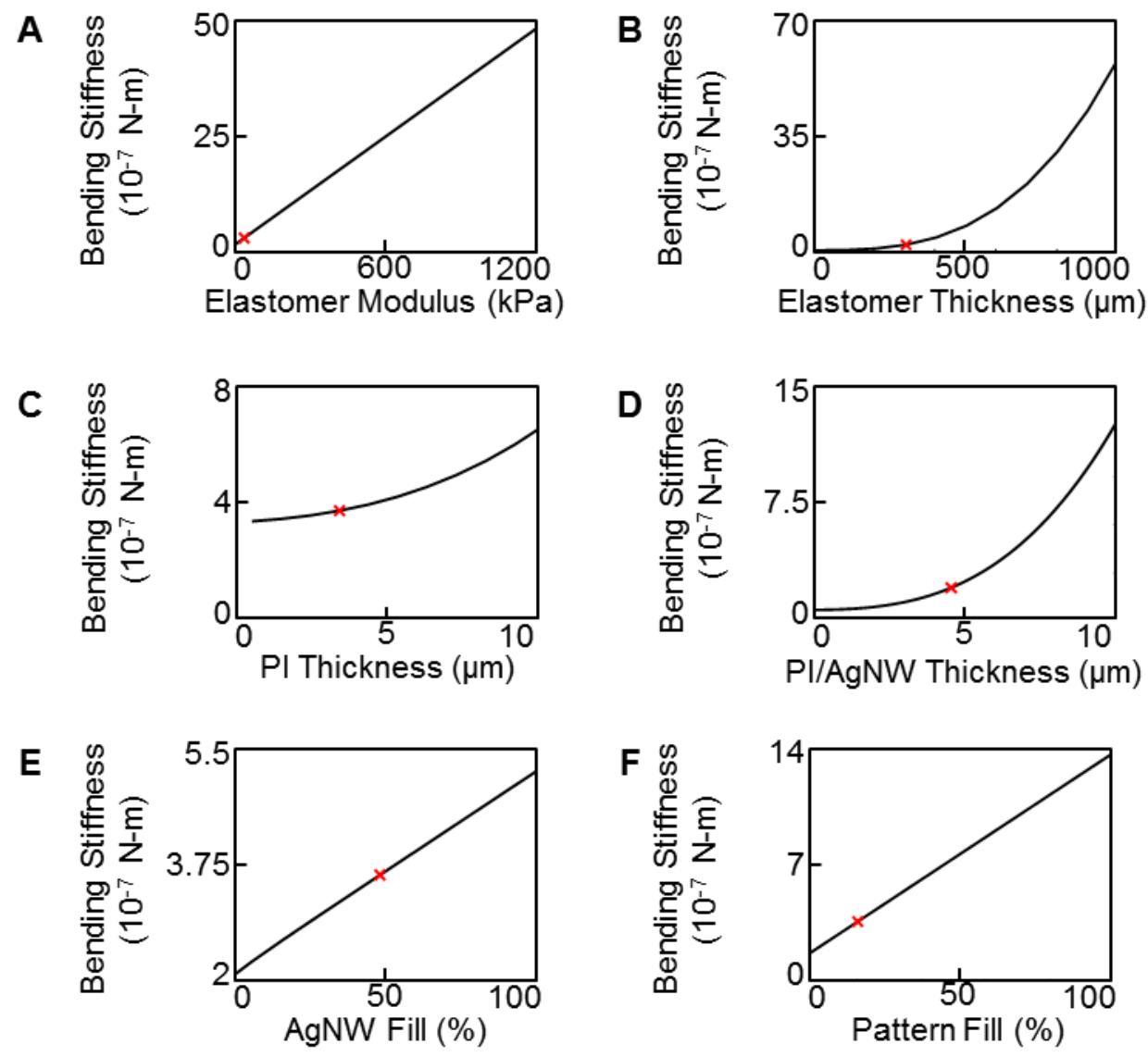

Figure S12. Effect on sensor bending stiffness of different sensor parameters: (A) elastomer modulus, (B) elastomer thickness, (C) PI thickness, (D) composite PI/AgNW thickness, (E) $\mathrm{AgNW}$ fill fraction of composite layer, and $(\mathrm{F})$ sensor pattern fill fraction. The red ' $\mathrm{X}$ ' indicates the value used in the human health monitoring sensor. 

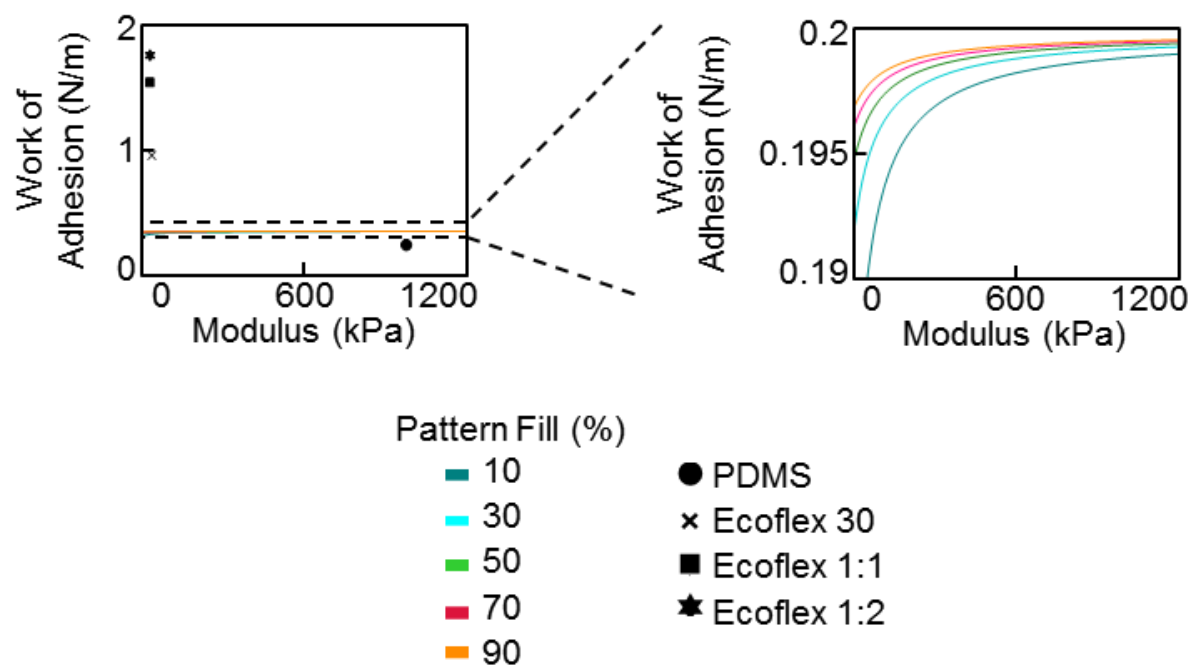

Figure S13. Conformal contact criteria for printed sensor based on elastomer encapsulation and AgNW/PI areal fraction. When elastomer properties are above the line thresholds for each areal fraction, conformal contact occurs. 

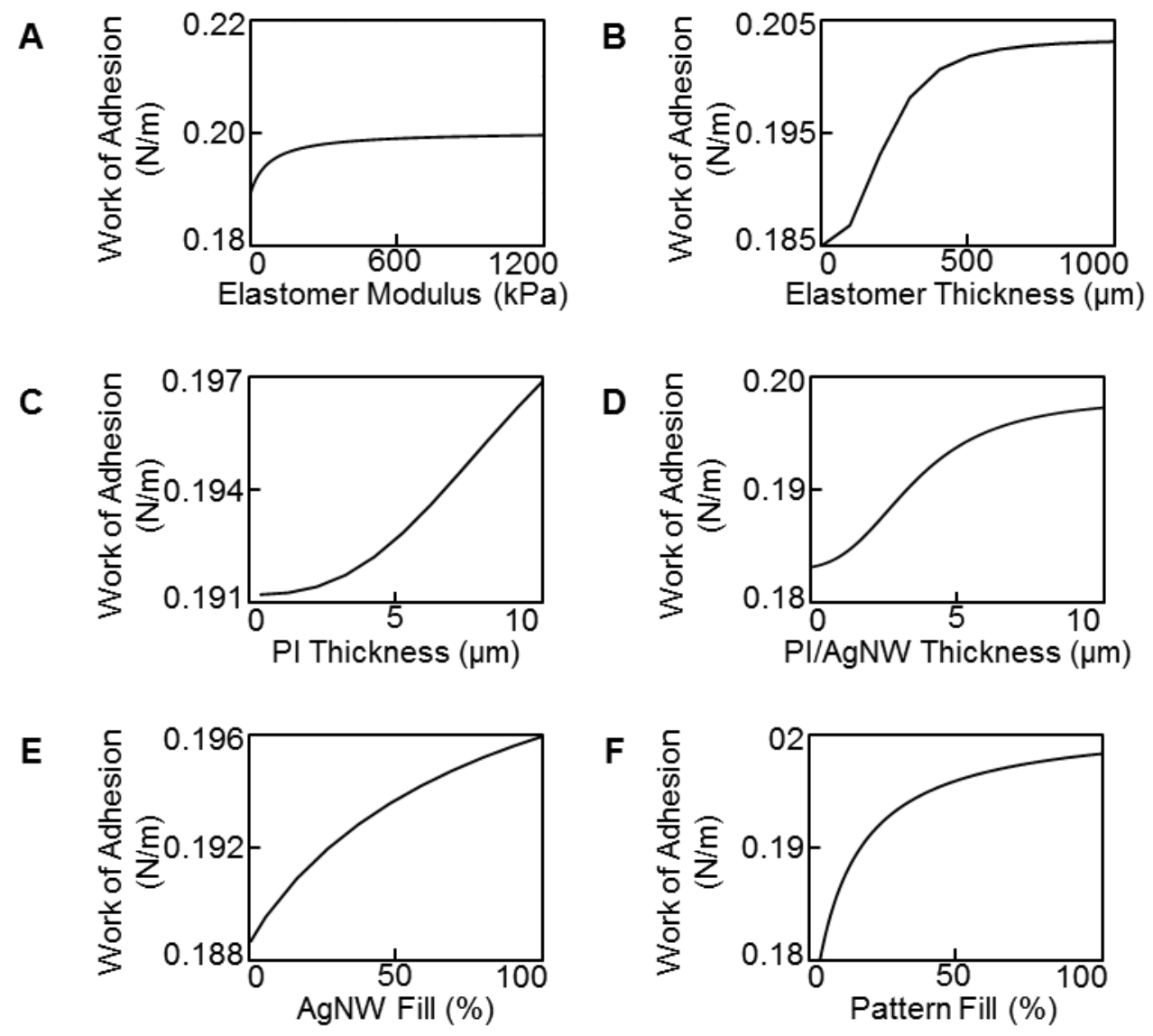

Figure S14. Effect on minimum work of adhesion required for conformal contact of different sensor parameters: (A) elastomer modulus, (B) elastomer thickness, (C) PI thickness, (D) composite PI/AgNW thickness, (E) AgNW fill fraction of composite layer, and (F) sensor pattern fill fraction. For the given parameter, if the work of adhesion is higher than the plotted line, then conformal contact is achieved. 


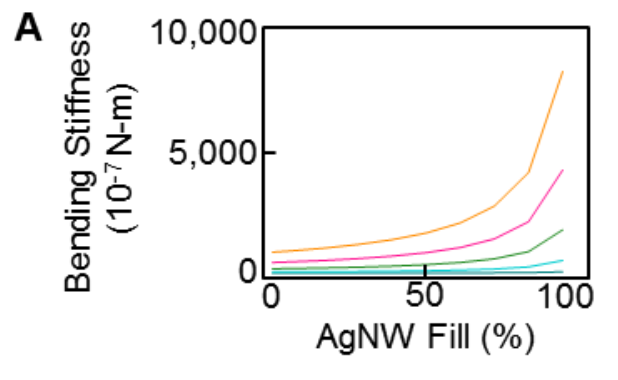

$$
\begin{gathered}
\text { Sensor } \\
\text { thickness }(\mu \mathrm{m}) \\
=200 \\
=400 \\
=600 \\
=800 \\
=1000
\end{gathered}
$$

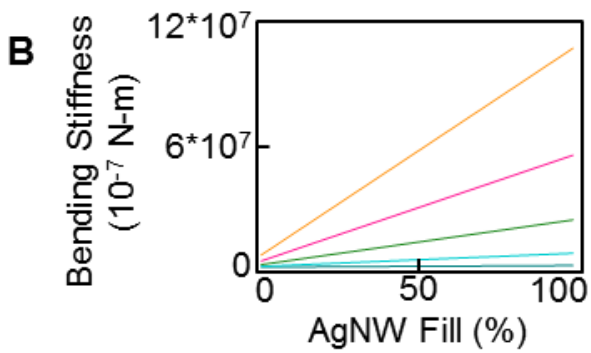

Figure S15. Estimated bending stiffness of a sensor using a PDMS-AgNW composite.

Different fill fractions and sensor thicknesses are shown. Bending stiffness calculated using (A) Reuss model and (B) Voigt model to determine composite stiffness as a lower bound and upper bound estimate, respectively. 


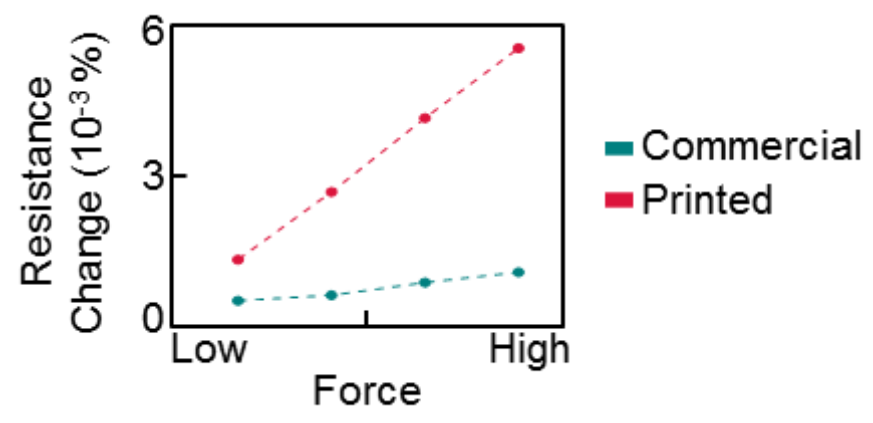

Figure S16. Comparison of force sensitivity between a commercial strain sensor and a printed flexible soft sensor. 

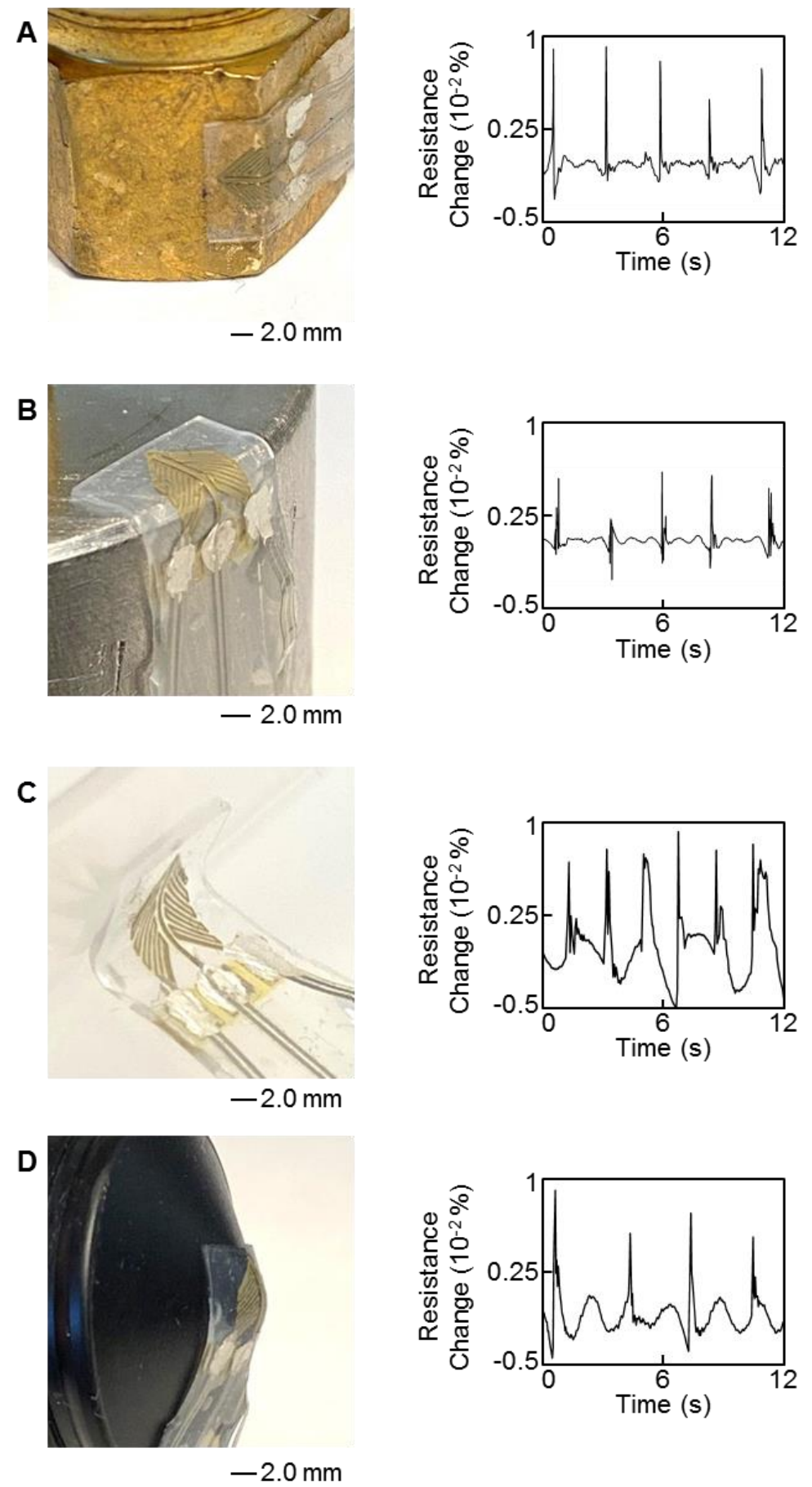

Figure S17. Printed strain sensor laminated on various surface geometries: (A) $60^{\circ}$ bend, (B) $90^{\circ}$ bending convex, (C) $90^{\circ}$ concave bending, and (D) cone shape. Resistance signals during impact are shown for each geometry. 


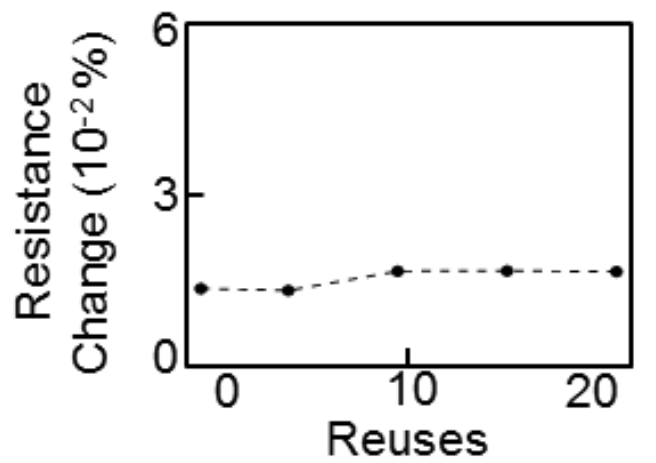

Figure S18. Average resistance change of sensor during impacts after multiple reuses. 

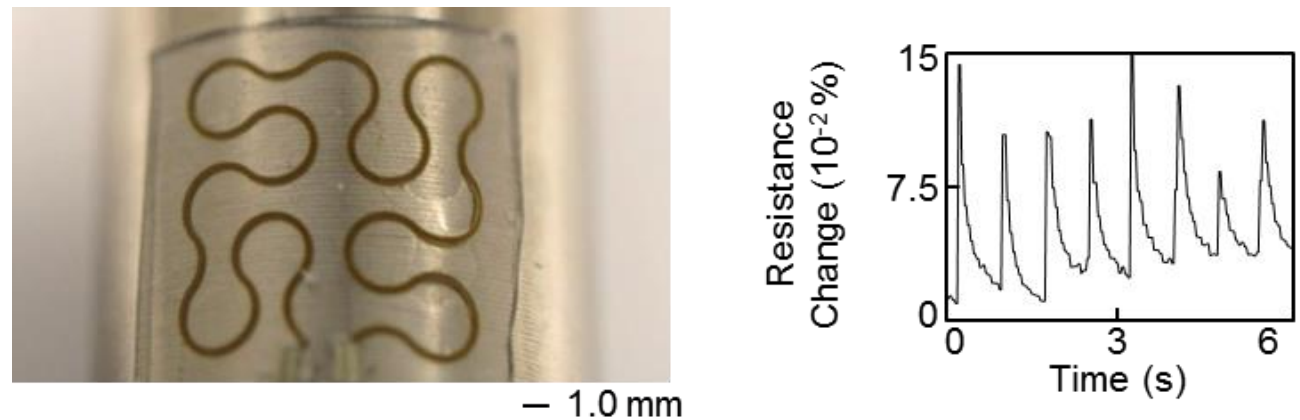

Figure S19. Stretchable sensor for structural monitoring. 

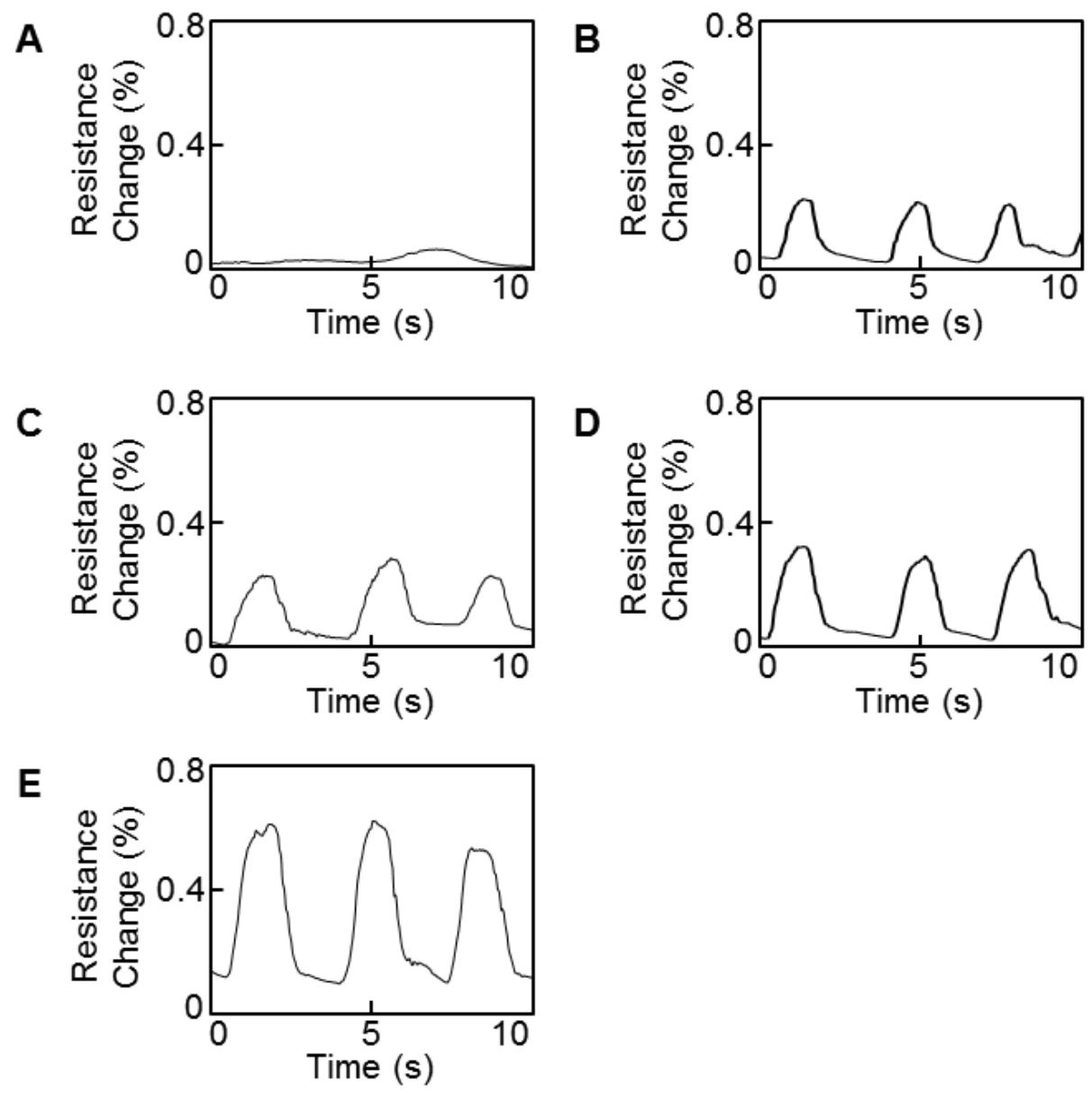

Figure S20. Resistance signals from bending finger at (A) $0^{\circ}$, (B) $30^{\circ}$, (C) $45^{\circ}$, (D) $60^{\circ}$, and (E) $90^{\circ}$. 
Table S1. Print parameters for PI and AgNW

\begin{tabular}{|c|c|c|}
\hline & PI & AgNW \\
\hline Ink Base & PI-2545 precursor & $1 \%$ AgNW in IPA \\
\hline Ink Solvent & NMP & IPA \\
\hline Atomizer & Pneumatic & Ultrasonic \\
\hline Sheath Rate $[\mathrm{ccm}]$ & 20 & 40 \\
\hline Atomization Rate $[\mathrm{ccm}]$ & 1470 (atomization) & 40 \\
\hline Nozzle Diameter $(\mu \mathrm{m})$ & 300 & 200 \\
\hline Printing Speed $[\mathrm{mm} / \mathrm{s}]$ & 10 & 2 \\
\hline Printing Passes & 4 & Variable \\
\hline Stage Temperature $\left[{ }^{\circ} \mathrm{C}\right]$ & 80 & N/A \\
\hline Curing Temperature $\left[{ }^{\circ} \mathrm{C}\right]$ & 240 & 80 \\
\hline
\end{tabular}


Table S2. Peel energy and modulus of elastomers.

\begin{tabular}{|c|c|c|c|}
\hline Material & $\begin{array}{c}\text { Peel Energy (mJ) } \\
\mathbf{1}^{\text {st }} \mathbf{u s e}\end{array}$ & $\begin{array}{c}\text { Peel Energy (mJ) } \\
\mathbf{1 0}^{\text {th }} \mathbf{u s e}\end{array}$ & Modulus (kPa) \\
\hline PDMS & 1.1 & 1.0 & 959.4 \\
\hline Ecoflex 30 & 0.6 & 0.6 & 53.7 \\
\hline Ecoflex 1:1 & 0.9 & 0.8 & 46.8 \\
\hline Ecoflex 1:2 & 1.1 & 1.1 & 47.2 \\
\hline Ecoflex Gel & 1.2 & 1.3 & 38.7 \\
\hline Silbione & 16.9 & 16.5 & 50.6 \\
\hline
\end{tabular}


Table S3. Peel energy of human health monitoring sensor from a steel surface and skin. The sensor used a 1:2 mixture of Ecoflex 30 and Ecoflex Gel.

\begin{tabular}{|c|c|c|}
\hline Surface & $\begin{array}{c}\text { Peel Energy (mJ) } \\
\mathbf{1}^{\text {st }} \mathbf{u s e}\end{array}$ & $\begin{array}{c}\text { Peel Energy (mJ) } \\
\mathbf{1 0}^{\text {th }} \text { use }\end{array}$ \\
\hline Steel & 1.1 & 1.1 \\
\hline Skin & 1.1 & 0.8 \\
\hline
\end{tabular}


Video S1. Aerosol jet printing of a soft strain sensor with polyimide and silver nanowires.

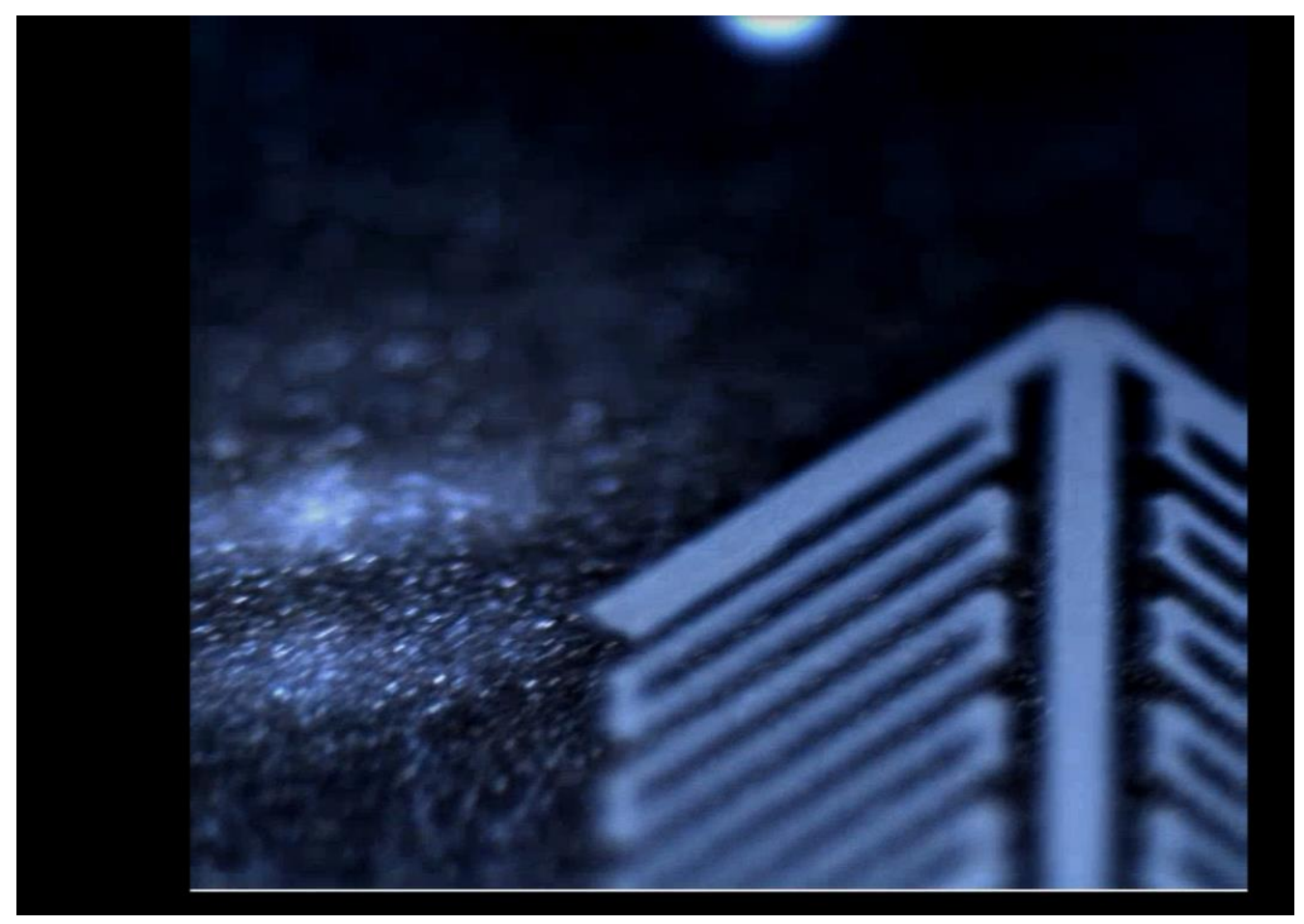




\section{References}

(1) Kim, Y. S.; Mahmood, M.; Lee, Y.; Kim, N. K.; Kwon, S.; Herbert, R.; Kim, D.; Cho, H. C.; Yeo, W. H. All-in-One, Wireless, Stretchable Hybrid Electronics for Smart, Connected, and Ambulatory Physiological Monitoring. Advanced Science 2019, 6 (17), 1900939.

(2) Yeo, W. H.; Kim, Y. S.; Lee, J.; Ameen, A.; Shi, L.; Li, M.; Wang, S.; Ma, R.; Jin, S. H.; Kang, Z. Multifunctional epidermal electronics printed directly onto the skin. Advanced Materials 2013, 25 (20), 2773-2778.

(3) Cuenot, S.; Frétigny, C.; Demoustier-Champagne, S.; Nysten, B. Surface tension effect on the mechanical properties of nanomaterials measured by atomic force microscopy. Physical Review B 2004, 69 (16), 165410.

(4) Dai, S.; Zhao, J.; He, M.-r.; Wang, X.; Wan, J.; Shan, Z.; Zhu, J. Elastic properties of GaN nanowires: Revealing the influence of planar defects on Young's modulus at nanoscale. Nano letters 2015, 15 (1), 8-15.

(5) Vivekchand, S.; Ramamurty, U.; Rao, C. Mechanical properties of inorganic nanowire reinforced polymer-matrix composites. Nanotechnology 2006, 17 (11), S344. 